\title{
Surgical Management of Type II Big Bubble in Deep Anterior Lamellar Keratoplasty
}

\author{
${ }^{1}$ Sandro Coscarelli, ${ }^{2}$ Leonardo Torquetti
}

\section{ABSTRACT}

Purpose: To present some simple surgical maneuvers to manage a type 2 big bubble (BB) in deep anterior lamellar keratoplasty (DALK).

Methods: Four DALK procedures with type 2 or mixed BB were performed. One was in the right eye of a 25 -year-old female. The corrected distance visual acuity (CDVA) was 20/200 due to advanced keratoconus. The second case was in a 28-year old male with central-anterior stromal scarring and neovascularization due to infectious keratitis and CDVA $<20 / 400$. The third case was in a 20 -year-old male with advanced keratoconus and CDVA $<20 / 400$. The fourth case was a 49 -year-old female, with high irregular astigmatism due to radial keratotomy and her CDVA was 20/150.

Results: At the last follow-up at 12 months, the first case of advanced keratoconus had a clear graft with CDVA $=20 / 50$ and some Descemet's folds that were in the visual axis, corresponding to the are of type $2 \mathrm{BB}$ that was not deflated intraoperatively. The second case achieved a CDVA $=20 / 40$, without folds at the visual axis at 12 months of follow-up. In the third and fourth patients with mixed BB where DL was retained, the CDVA was $20 / 25$ and $20 / 30$, respectively. No intraoperative DM perforation or postoperative complications were noted.

Conclusion: Type 2 BB in DALK can be properly managed in order to avoid perforation and to attain a good visual outcome.

Keywords: Deep anterior lamellar keratoplasty, Dua's layer, Type II big bubble.

How to cite this article: Coscarelli S, Torquetti L. Surgical Management of Type II Big Bubble in Deep Anterior Lamellar Keratoplasty. Int J Kerat Ect Cor Dis 2018;7(2):145-150.

\section{Source of support: Nil}

\section{Conflict of interest: None}

Ethical approval: All procedures performed in studies involving human participants were in accordance with the ethical standards of the institutional board of Ennio Coscarelli Eye Clinic and with the 1964 Helsinki declaration and its later amendments or comparable ethical standards.

Informed consent: Informed consent was obtained from all individual participants included in the study.

\footnotetext{
1,2 Head

${ }^{1}$ Ennio Coscarelli Eye Clinic, Belo Horizonte, Brazil

${ }^{2}$ Center of Excellence in Ophthalmology, Pará de Minas, Brazil

Corresponding Author: Sandro Coscarelli, Head, Ennio Coscarelli Eye Clinic, Belo Horizonte, Brazil, e-mail: sandrocoscarelli@gmail.com
}

\section{INTRODUCTION}

Deep anterior lamellar keratoplasty (DALK) is a surgical procedure to replace a scarred or irregular stroma while preserving the host endothelium with minimal effect on endothelial cell density and eliminating the risk of endothelial graft rejection. ${ }^{1,2}$ The visual acuity (VA) after DALK is similar to VA after penetrating keratoplasty (PK), ${ }^{2,3}$ and better than anterior lamellar keratoplasty. DALK also confers an additional advantage of better globe security compared to PK.

Originally, DALK was performed using manual stromal dissection. ${ }^{4}$ The greatest drawback of early DALK techniques was significant residual stroma was retained, which provided a platform for vascular ingrowth, led to a degree of postoperative scarring in the interface and at times some of the original pathologies was retained in the residual stroma. Also, the rough dissection plane could impair vision. The main disadvantages of DALK are the significant learning curve in mastering the technique and that it is a laborious procedure. Postoperative astigmatism is similar to that after PK.

Anwar and Teichmann's ${ }^{5}$ big-bubble technique of injecting air into the corneal stroma to separate Descemet's membrane, significantly improved DALK outcomes. Complete removal of the superficial and deep stroma created a clear graft interface with minimal irregularities. The visual result is excellent and postoperative interface problems from vascularization or scarring of the recipient corneal bed are insignificant.

Although this technique is reproducible and rates of successful big-bubble formation as high as $80-90 \%$ have been described, ${ }^{6,7}$ it has a steep learning curve and success rates can be lower for beginners.

When air is injected into the corneal stroma three types of BB can be achieved. ${ }^{8}$

1. Type $1 B B$ : The air detaches the Dua's layer (DL) from the deep stroma forming a large central bubble. This is the desired type of bubble in DALK, as DL presents additional force to the recipient cornea. After removing the receiver stroma, the complex of host DL and $\mathrm{DM}$ and endothelial cells is preserved.

2. Type $2 \mathrm{BB}$ : The $\mathrm{DM}$ is separated from the posterior side of DL by the injected air. This BB is bigger with a thinner wall made of DM and endothelial cells only. 
3. Mixed BB: The above two coincide, usually type 1 is complete and type 2 is incomplete, but both can be complete. About $80-85 \%$ of the BB are of type 1 and $15-20 \%$ of type 2 or mixed. When the DL level is mechanically opened during DALK, the host's preserved tissue complex is often as described above with a type $1 \mathrm{BB}$. The surface of this tissue composite gives a coarse look related to fragmented components or fibers of collagen that are overextended when DL is detached from the deep stroma. These fibers can be seen to extend between the posterior side of the deep stroma and the anterior surface of DL during mechanical separation and have to be disconnected or cut. In comparison, the surface of the DM (type $2 \mathrm{BB}$ ) appears very smooth and dull. ${ }^{9}$ It is imperative to differentiate the type of BB obtained. DALK can be successfully completed with any type of BB but the type $2 \mathrm{BB}$ is weak and predisposed to ripping or bursting intraoperatively, imposing conversion to PK.

We present some simple surgical maneuvers to manage type 2 and mixed big bubbles in deep anterior lamellar keratoplasty, in order to avoid complications and attain a good visual outcome.

\section{MATERIALS AND METHODS}

Five DALK procedures with type 2 or mixed BB were performed. All surgeries were done under peribulbar block by the same surgeon (SC) at the Ennio Coscarelli Eye Clinic, Belo Horizonte, Brazil. One was in the right eye of a 25 -year-old female. The corrected distance visual acuity (CDVA) was 20/200 due to advanced keratoconus. The second case was in a 28-year-old male with centralanterior stromal scarring and neovascularization due to infectious keratitis and CDVA $<20 / 400$. The third case was in a 20-year-old male with advanced keratoconus and CDVA $<20 / 400$. The fourth case was a 49 -year-old female, with high irregular astigmatism due to radial

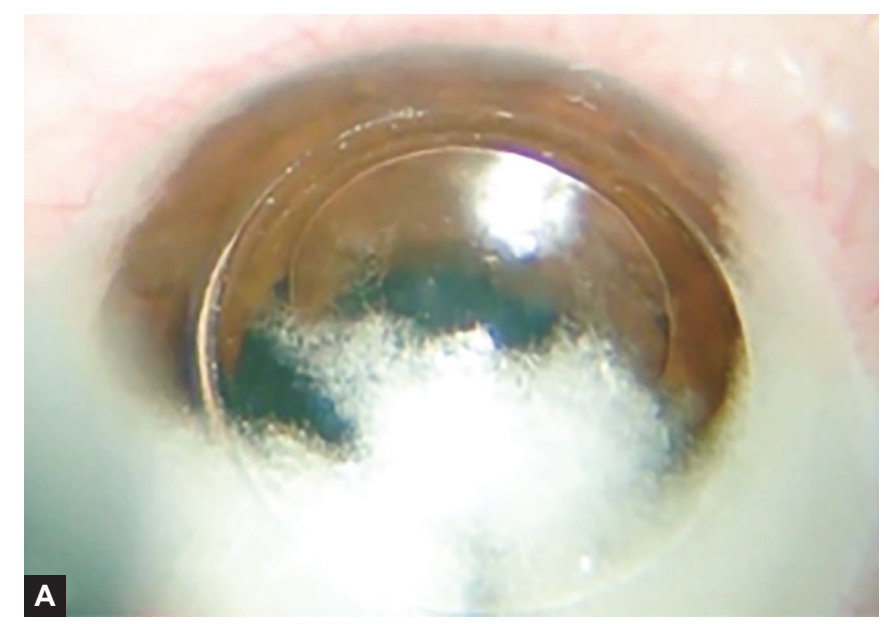

Figs 1A and B: (A) Type 2 big bubble; (B) Deeper stromal dissection around the bubble

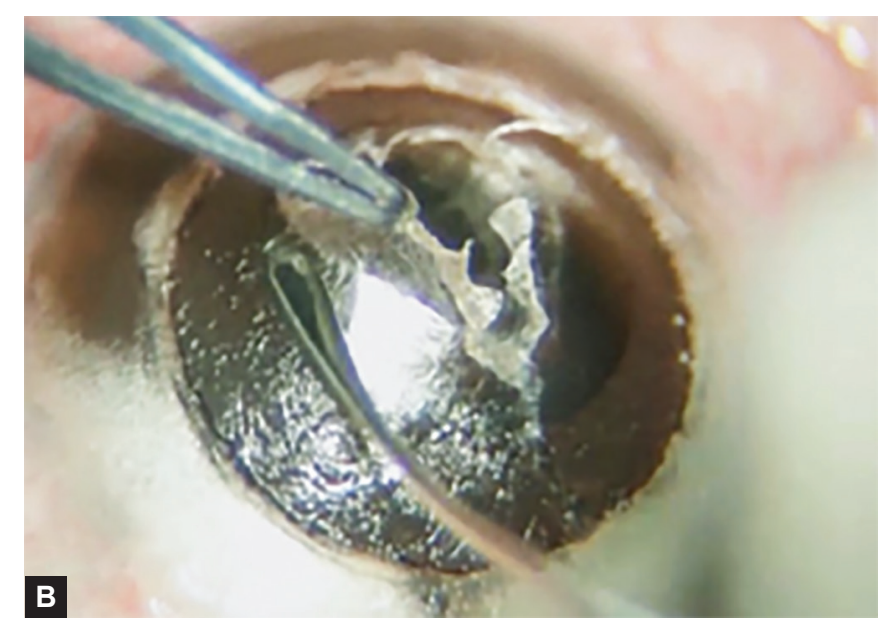

keratotomy and her CDVA was 20/150. The fifth case was a 30-year-old female with very advanced keratoconus in both eyes. The CDVA was $<20 / 400$ in both eyes.

\section{Surgical Technique}

The surgical procedure was performed under peribulbar anesthesia. Initially, partial-thickness trephination of $8 \mathrm{~mm}$ was performed. We used a diamond knife set at $90 \%$ of corneal depth $\left(90^{\circ}\right.$ meridian), to make a $0.9 \mathrm{~mm}$ radial incision close to the limbus and a corneal pocket was created using the Ferrara spreader (AJL Ophthalmic, Vitoria, Spain). ${ }^{10}$ Air was injected with a cannula inserted through the pocket, radially to the center of cornea to create a BB. In all these cases we obtained a type 2 or mixed BB. Blunt-tipped Vannas scissors was used to remove the anterior stromal (half to two-thirds thickness) tissue by cutting along the trephination groove.

The first case with advanced keratoconus had a type $2 \mathrm{BB}$ (Fig. 1A). A paracentesis was performed and the anterior stroma (half to two-thirds thickness) was removed. Thereafter, a limited deeper stromal dissection was performed with a blunt dissector (Fig. 1B). Instead of entering the BB to deflate it we decided to leave the type $2 \mathrm{BB}$ with a layer of deep stroma anterior to it (Supplementary Video 1). As the type 2 BB was not drained, postoperatively after its absorption some Descemet stria was found in the visual axis.

In the second case, a patient with a scar and neovascularization secondary to infectious keratitis, a type 2 $\mathrm{BB}$ was formed and extended to the corneal periphery in the inferior half (Fig. 2A). A deep dissection with a blunt spatula was performed. A needle was used to gain entry into the bubble (Fig. 2B). The type $2 \mathrm{BB}$ was deflated in order to avoid Descemet folds. The superficial stroma was dissected and excised. A subsequent very gentle blunt dissection was done to deflate the bubble and subsequently excise the deep stroma leaving only 

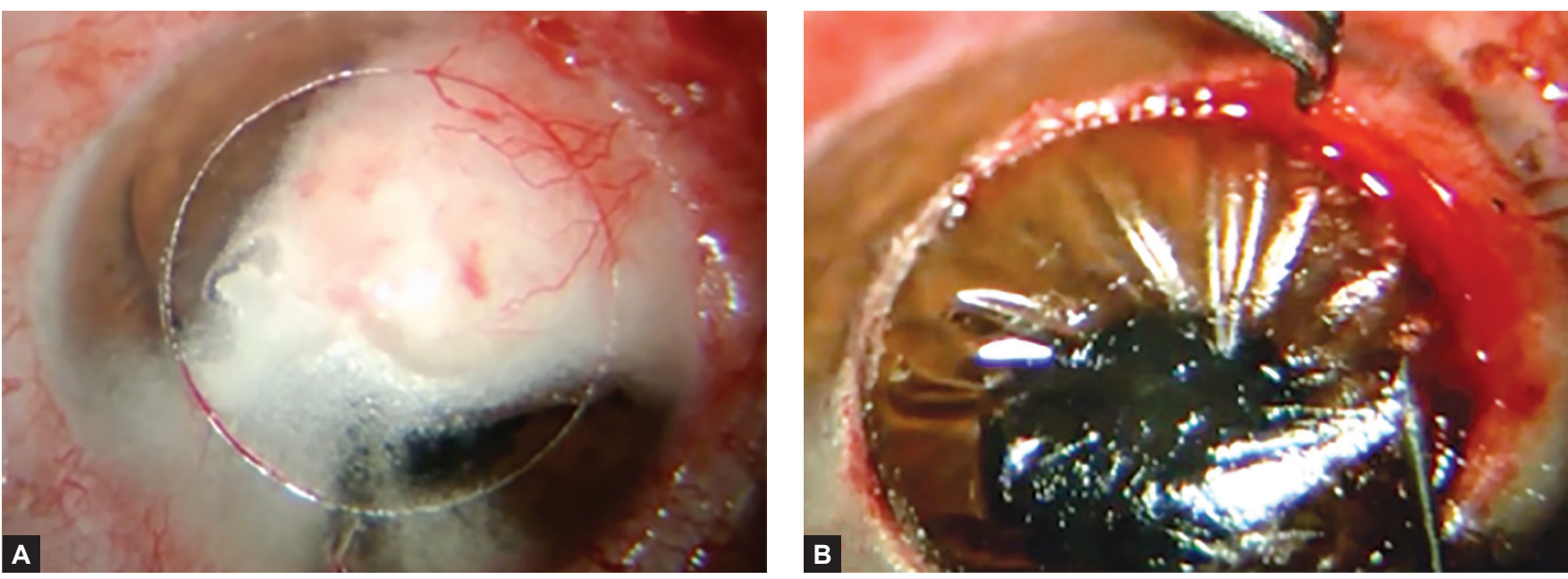

Figs 2A and B: (A) Type 2 BB in the periphery; (B) Deflating the bubble. Note the striae in the tissue, which appears with pressure of the needle tip
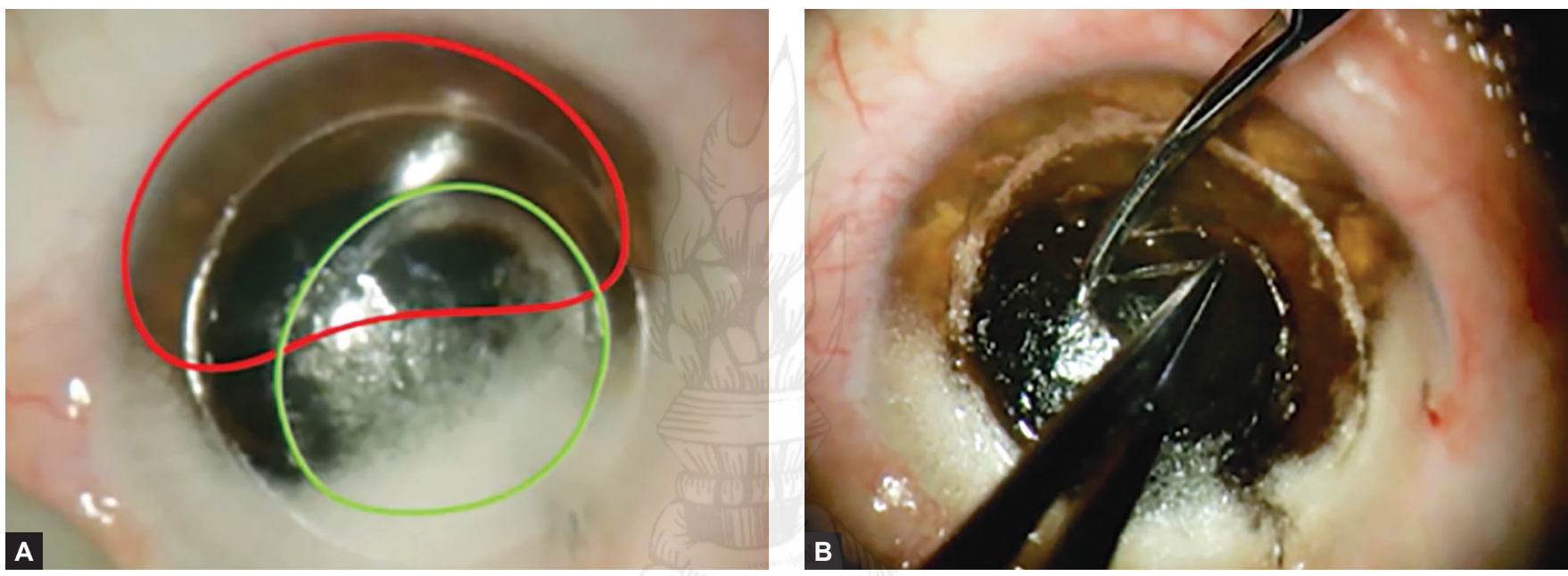

Figs 3A and B: (A) Mixed big bubble: Type 1 (green outline) and 2 big bubble (red outline) are seen;

(B) Dua's layer dissection in the visual axis

the Descemet's membrane exposed. While excising the deep stroma, one tip of a blunt tipped corneal scissors was introduced in the plane between DL and DM and the deep stroma was lifted anteriorly on the edge of the blade before cutting along the trephination groove. (Supplementary Video 2).

The third case, a keratoconus patient previously implanted with an intrastromal corneal ring segment (ICRS) had a mixed BB (Fig. 3A). A paracentesis was performed, followed by superficial stromal dissection. Entry into the type 1 BB was made with a 15-degree knife. Dissection of the deep stroma and removal of ICRS lead to communication with the type $2 \mathrm{BB}$ through the DL. This caused the type $2 \mathrm{BB}$ to deflate. The deep stroma corresponding to the area of the type $1 \mathrm{BB}$ was excised exposing DL in the visual axis (Fig. 3B).

The fourth case, a patient with high irregular astigmatism due to radial keratotomy, had a mixed BB (Fig. 4A). Superficial stromal dissection was performed followed by paracentesis, and then entry into the type
1 BB was made with a 15-degree knife. During dissection and excision of the deep stroma, communication occurred through DL with the type $2 \mathrm{BB}$, which partially collapsed. The remainder of the type $2 \mathrm{BB}$ was left untouched with exposed Dua's layer (Fig. 4B) (Supplementary Video 3).

The fifth case, a patient with very advanced keratoconus (Fig. 5A), had a type 2 BB in both eyes (Fig. 5B). The superficial and deep stroma were dissected and excised. An anterior chamber paracentesis was done to reduce the intraocular pressure to allow safe entry into the type 2 BB (Fig. 5C). In the first operated eye (left eye) an incomplete dissection was done in the optical zone compromising the visual axis. After 30 days, a reoperation was done in order to increase the optical zone dissection. In the right eye of the same patient, the type $2 \mathrm{BB}$ was deflated, completely removing the residual stromal and Dua's layer (Supplementary Video 4).

In all cases the donor cornea was perforated out from the endothelial side, oversized by $0.5 \mathrm{~mm}$ in comparison to receiver trephination. The graft endothelium was 


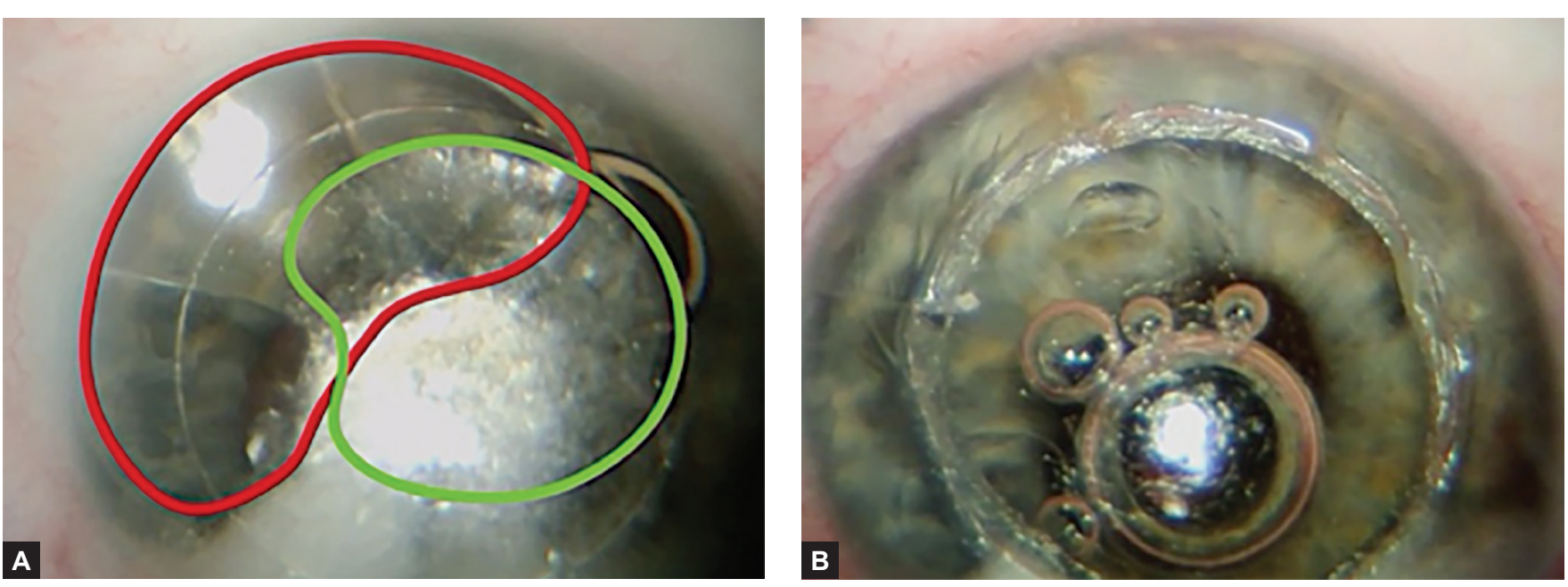

Figs 4A and B: (A) Mixed big bubble: Type 1 (green outline) and 2 big bubble (red outline) are visiblel; (B) Small type 2 big bubble left untouched (red arrow)

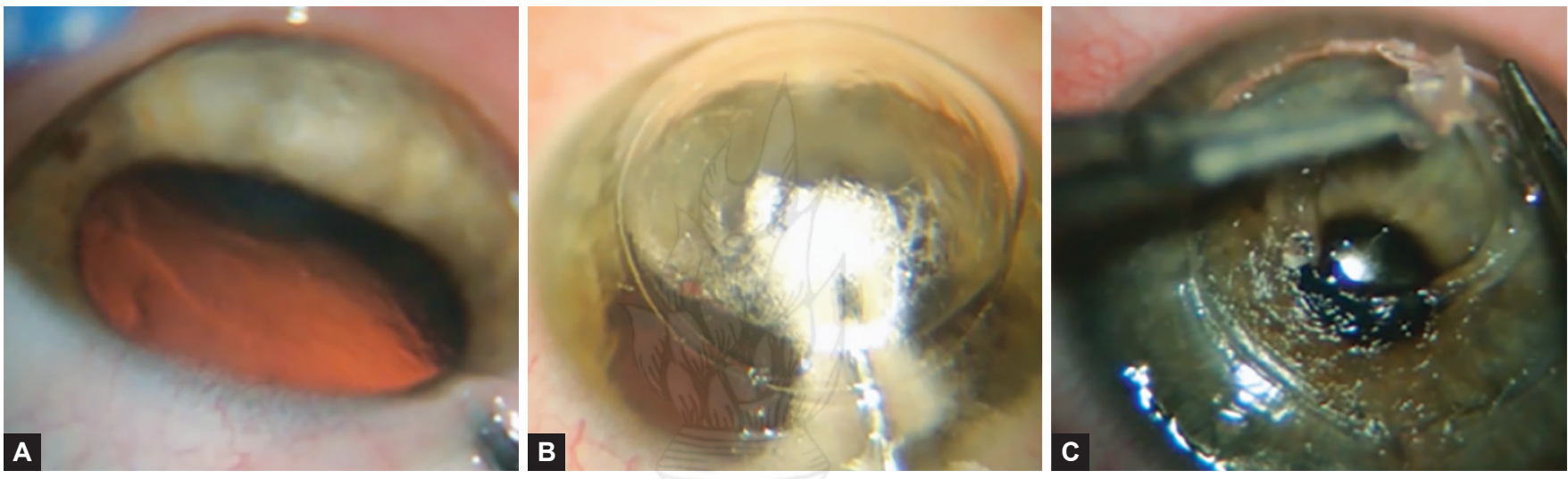

Figs 5A to C: (A) Advanced keratoconus. Profile intraoperative view; (B) The outline of type 2 big bubble is seen with little stromal emphysema around the needle tip; (C) Bare Descemet membrane, showing a shiny smooth appearance

totally removed. The graft was sutured in place using uninterrupted or 16 interrupted 10-0 nylon sutures

Postoperative prescription included moxifloxacin and dexamethasone four times a day for a week and tapered over a period of 6 weeks. Lubricants were used several times a day. The sutures were removed 12 months postsurgery.

\section{RESULTS}

At the last follow-up at 12 months, the first case of advanced keratoconus had a clear graft with CDVA =
20/50 (Table 1) and some Descemet's folds that were in the visual axis (Fig. 6), corresponding to the are of type 2 BB that was not deflated intraoperatively. The second case (scar after keratitis) achieved a CDVA $=20 / 40$, without folds at the visual axis at 12 months of follow-up. In the third and fourth patients with mixed BB where DL was retained, the CDVA was 20/25 and 20/30, respectively. No intraoperative DM perforation or postoperative complications were noted.

Table 1: Preoperative and postoperative data on CDVA

\begin{tabular}{lllll}
\hline & & & \multicolumn{2}{c}{ CDVA } \\
\cline { 4 - 5 } Cases & Diagnosis & BB type & Preoperative & Postoperative \\
\hline 1 & Keratoconus & 2 & $20 / 200$ & $20 / 50$ \\
2 & Scar after keratitis & 2 & $<20 / 400$ & $20 / 40$ \\
3 & Keratoconus & Mixed & $<20 / 400$ & $20 / 25$ \\
4 & Irregular astig after RK & Mixed & $20 / 150$ & $20 / 30$ \\
5 & Keratoconus & 2 & $<20 / 400$ & $20 / 25$ \\
\hline
\end{tabular}

CDVA, corrected distance visual acuity, RK, radial keratotomy 


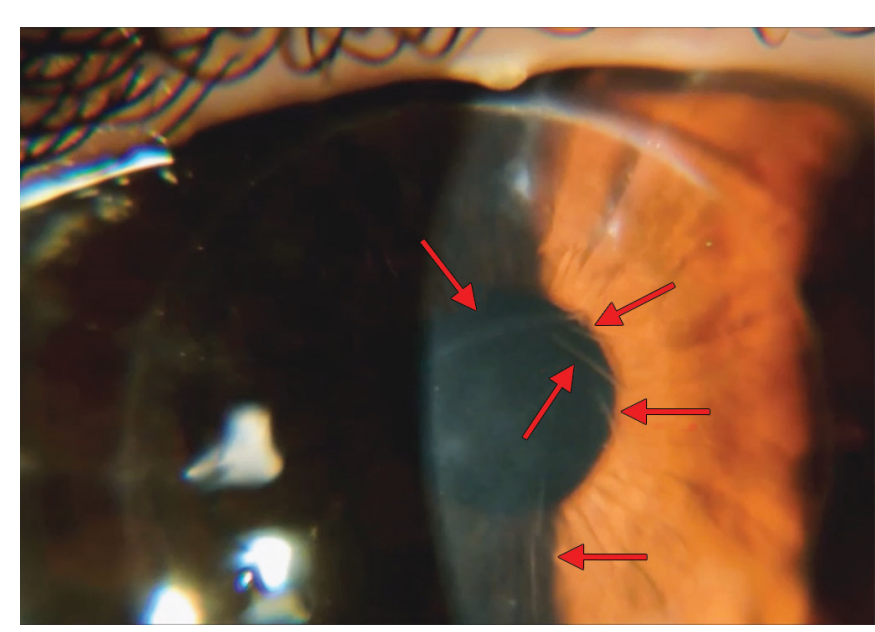

Fig. 6: Descemet's folds (arrows) are seen in the cornea postoperatively

\section{DISCUSSION}

One of the major intraoperative problems of BB DALK is perforation of DM or the DM+DL composite. Perforation of the latter in a type 1BB is usually in the form of a microperforation, which is generally not a problem. However, perforation of the DM in type 2 or mixed BB is usually a macro perforation or a burst, necessitating conversion to PK. The type $2 \mathrm{BB}$ is considered an unusual occurrence during DALK. It is more prone to form in old patients and those with deep corneal scars, advanced keratoconus, and thin corneas. ${ }^{11}$

In one study, eyes with the type 2 bubble showed a high frequency of conversion to PK (86\%). ${ }^{11}$ Because of this, it is imperative that the surgeon is able to recognize intraoperatively which BB has formed and better surgical strategies adopted to manage type 2 bubbles for successful DALK.

Dua et al. ${ }^{8}$ have shown a pre-Descemet's layer [Dua's layer (DL)] which was described by the authors to be much stronger and robust than DM and could resist a pressure of approximately $700 \mathrm{~mm}$ of $\mathrm{Hg}$.

The source of the bubble at the middle and its centrifugal increase, the adjacent white ring, its diameter extending to no more than $8.5-9 \mathrm{~mm}$ and surface 'coarseness' related to broken filaments of collagen prolonging between DL and deep stroma, are signs of a type 1 BB. ${ }^{9}$ The 'coarse' appearance of the anterior surface of the DL in a type $1 \mathrm{BB}$ is clearly perceptible after removal of the deep stroma. With a type $1 \mathrm{BB}$ a light effort at the insertion of a blunt spatula directed centrifugally towards the limbus, between the surface of DL and the deep stroma along the perimeter of the trephine cut will be encountered with resistance but not with a type $2 \mathrm{BB}$ where the plane spreads further near or up to the limbus. However, this maneuver is dangerous and should be tried with caution. A type 2 BB expands very quickly, starts at the periphery or in the central area in advanced keratoconus and spreads centrally covering an area much larger than the trephine mark, often to the periphery of the cornea. It has a very smooth surface with no 'coarseness' as seen with a type $1 \mathrm{BB}$. Furthermore, the type 2 BB extends centrifugally to the periphery of the cornea. ${ }^{8,12}$ The tip of the paracentesis needle or knife can easily rupture the bubble if it makes contact with the bubble wall. This can be avoided by making a very peripheral paracentesis with a type 2 BB before stabbing the roof of the bubble. Thereafter, once the air has escaped and the bubble has emptied, the paracentesis can be enlarged.

The main measures in dealing with type $2 \mathrm{BB}$, as described in this paper are (a) leave the type 2 BB if there is a high risk of perforation and the bubble is not in the visual axis, avoiding the plane between DL and $\mathrm{DM}$. This was done in the first patient where a thin layer of deep stroma was retained; (b) decompression of the anterior chamber before entry into the type $2 \mathrm{BB}$. This is an important step. Intrastromal air injection and creation of a $\mathrm{BB}$ cause the intraocular pressure to rise. Hence, when the $\mathrm{BB}$ is deflated, the DM or DM + DL composite moves anteriorly very rapidly and can make contact with the knife tip causing a perforation. Paracentesis reduces the pressure very satisfactorily; (c) The excision of the anterior wall of a type- $2 \mathrm{BB}$ can be limited to the visual axis. The DM is very fragile; therefore, any attempt to dissect up to periphery may increase the risk of perforation or tear, and (d) minimize contact of the tip of the corneal scissors with DM during excision of the deep stroma by lifting the stroma anteriorly on the edge of the blade of the scissors before cutting.

When a mixed BB is achieved, the DM is protected by the DL anterior to it with a cushion of air in between, which is usually partial. Once the deep stroma is excised the type $1 \mathrm{BB}$ is emptied. The DL anterior to the type 2 $\mathrm{BB}$ can be punctured with a 30-gauge needle to deflate the type $2 \mathrm{BB}$. This effectively makes the resultant situation akin to that of a type $1 \mathrm{BB}$. One could consider the injection of an air bubble in the anterior chamber at the end of the procedure. These tamponades the DM against the DL in the area where a type $2 \mathrm{BB}$ had formed. This is more important if there is a microperforations in the DM. In cases where the deep stroma and DL are likely to be affected by previous disease or surgery/injury as in our cases with scarring related to infectious keratitis and with and ICRS, the DL with the deep stroma could tear and result in spontaneous deflation of the type 2 BB. When deliberate deflation of the type 2 components of a hybrid BB is needed, it should be attempted through normal looking DL/deep stroma away from the area affected by scarring.

The CDVA seems to be the same with type 1 and type $2 \mathrm{BB}$, i.e., whether the interface of the transplanted 
cornea is with DL or DM but is likely to be not as good if deep stroma is retained anterior to DL as in our first case. However, this aspect of DALK, especially the quality of vision after types 1 and 2 BB needs further study, notwithstanding the greater risk of DM tear and switch to PK with a type $2 \mathrm{BB}$. Deflating the type $2 \mathrm{BB}$ is justifiable in cases of deep opacities or irregularities in pre-Descemet area, which can impair the visual acuity.

It is difficult to guess which type of BB will be accomplished through some procedures such as placing the needle/cannula tip in the deep stroma in the center of the cornea help in achieving a type $1 \mathrm{BB}$. Sarnicola et al. ${ }^{13}$ recently showed that the use of a flat cannula during the DALK procedure produces a statistically higher percentage of 'Descemetic DALK' (type $1 \mathrm{BB}$ ) and makes the maneuver more controllable compared with air injection with a needle. Their study was published previous to the identification of the diverse planes of cleavage in the different types of BB. They had used the term 'Descemetic DALK' to describe the situation when a BB was achieved and a 'pre-Descemetic DALK' when a BB was not attained and manual dissection to deep stromal depth was required. In the latter situation, some deep stromal tissue was retained.

With type $2 \mathrm{BB}$, we suggest that it is not essential to excise the deep stroma along the trephine mark. This could be done only in the visual axis retaining a wide rim of deep stroma, which would confer additional strength to the eye reduce risk of DM tear as less manipulation is required.

Though type 2 BB seems to be more common in advanced keratoconus, after radial keratotomy and in case of deep scars and in the elderly, ${ }^{10}$ it is seen in only $15-20 \%$ of cases of DALK. In these cases, despite the higher risk of perforation, successful DALK can be completed if the strategies suggested in this paper are adopted. In cases of previous hydrops, the big bubble should not be tried due to the rupture of Descemet caused by this condition.

\section{REFERENCES}

1. Sarnicola V, Toro P, Sarnicola C, et al. Long-term graft survival in deep anterior lamellar keratoplasty. Cornea 2012;31(6):621626.

2. Krumeich JH, Knulle A, Krumeich BM. Deep anterior lamellar (DALK) vs. penetrating keratoplasty (PKP): a clinical and statistical analysis. Klin Monbl Augenheilkd 2008;225(7):637648.

3. Reinhart WJ, Musch DC, Jacobs DS, et al. Deep anterior lamellar keratoplasty as an alternative to penetrating keratoplasty a report by the American Academy of Ophthalmology. Ophthalmol 2011;118(1):209-218.

4. Espandar L, Carlson AN. Lamellar keratoplasty: A literature review. J Ophthalmol 2013:894319.

5. Anwar M, Teichmann KD. Big-bubble technique to bare Descemet's membrane in anterior lamellar keratoplasty. J Cataract Refract Surg 2002;28(3):398-403.

6. Jafarinasab MR, Rahmati-Kamel M, Kanavi MR, et al. Dissection plane in deep anterior lamellar keratoplasty using the big-bubble technique. Cornea2010;29(4):388-391.

7. Javadi MA, Mohammad-Rabei H, Feizi S, et al. Visual outcomes of successful versus failed big-bubble deep anterior lamellar keratoplasty for keratoconus. J Ophthalmic Vis Res 2016;11(1):32-36.

8. Dua HS, Faraj LA, Said DG, et al. Human Corneal Anatomy Redefined. Ophthalmol 2013;120(9):1778-1785.

9. Katamish D, Faraj S. Differentiating type 1 from type 2 big bubbles in deep anterior lamellar keratoplasty. Clinical Ophthalmol 2015:9:1155-1157.

10. Coscarelli S, Torquetti L. Easy Bubble: Pachymetry-assisted Big-Bubble Deep Anterior Lamellar Keratoplasty. J Emmetrop 2012;3:153-156.

11. Goweida MB. Intraoperative review of different bubble types formed during pneumodissection (big-bubble) deep anterior lamellar keratoplasty. Cornea 2015;34(6):621-624.

12. Dua HS, Faraj LA SD. Dua's layer: its discovery, characteristics and clinical applications. Biomechanica y arquitetura corneal (corneal architecture and biomechanics). Ángeles del Buey Sayas MA, Cris Peris Martínez CP Elsevier 2014; Chapter 4 p 35-4747.

13. Sarnicola E, Sarnicola C, Sabatino F, et al. Cannula DALK Versus Needle DALK for Keratoconus. Cornea 2016; 35(12):1508-1511. 